(2) Open Access Full Text Article

ORIGINALRESEARCH

\title{
Dyspnea During Night-Time and at Early Morning in Patients with Stable COPD is Associated with Supine Tidal Expiratory Flow Limitation
}

This article was published in the following Dove Press journal:

International Journal of Chronic Obstructive Pulmonary Disease

\author{
Silvia Uccelli ${ }^{1}$ \\ Laura Pini ${ }^{\prime}$ \\ Damiano Bottone (iD ${ }^{\prime}$ \\ Piera Ranieri ${ }^{2}$ \\ Nicla Orzes' \\ Claudio Tantucci ${ }^{1}$ \\ 'Respiratory Medicine Unit, Department \\ of Experimental and Clinical Sciences, \\ University of Brescia, Brescia, Italy; \\ ${ }^{2}$ Department of Internal Medicine and \\ Rehabilitation, Istituto Clinico Sant'Anna, \\ Brescia, Italy
}

Rationale: Patients with chronic obstructive pulmonary disorder (COPD) complain of various COPD-related symptoms with different daily frequencies. During the night-time and at early morning, dyspnea is often reported and may predict an increased risk of COPD exacerbation and hospitalization and all-cause mortality. The aim of the study was to assess the underlying mechanisms of this symptom, seeking functional biomarkers of its occurrence.

Methods: Stable COPD patients with moderate-to-severe airflow obstruction and without confounding comorbidities underwent extensive baseline function respiratory tests. Spirometry, maximal flow-volume curves, lung volumes, and lung diffusion capacity parameters were obtained. Inspiratory capacity was also measured both in seated and supine positions. Forced oscillation technique (FOT) and negative expiratory pressure (NEP) method were used to establish the presence of tidal expiratory flow limitation (EFL) during recumbency. Questionnaires for recording COPD-related symptoms were administered. Sleep-related disturbances reported by the patients were also registered.

Results: Forty-two consecutive COPD patients aged $65 \pm 9$ completed the protocol. They were divided, according to the absence (NFL) or presence (FL) of supine EFL, in NFL group $(\mathrm{n}=17)$ and FL group $(\mathrm{n}=25)$. FL COPD patients had more severe airflow obstruction $\left(\mathrm{FEV}_{1}=\right.$ $46.4 \pm 19.4$ vs $65.1 \pm 12.5 \%$ pred., $\mathrm{p}<0.01)$ and they showed no increase of supine IC in contrast with NFL COPD patients $(\Delta \mathrm{IC}=0.080 \pm 0.18$ vs $0.390 \pm 0.28 \mathrm{~L}, \mathrm{p}<0.01)$. Dyspnea either during night-time and at early morning was significantly more reported in FL COPD patients than in NFL COPD patients $(\mathrm{p}<0.05)$ and in those with less than $10 \%$ increase in supine IC $(\mathrm{p}<0.05)$.

Conclusion: Supine EFL is frequently associated with both night-time and early morning dyspnea, suggesting that the development of recumbent dynamic pulmonary hyperinflation, heralded by the lack of increment of IC in supine position, is a pivotal mechanism of this symptom. No or trivial increase in supine IC may indicate the occurrence of dyspnea under these conditions.

Keywords: COPD, expiratory flow limitation, pulmonary hyperinflation, dyspnea, nocturnal symptoms, symptoms in the early morning, inspiratory capacity

\section{Introduction}

It has been recently recognized that in most patients suffering from Chronic Obstructive Pulmonary Disorder (COPD) their chronic symptoms may distribute differently and with different severity along the 24 hours of the day, being present throughout the day-time or during the night-time or also occurring at early morning
Correspondence: Laura Pini

Email laura.pini@unibs.it
International Journal of Chronic Obstructive Pulmonary Disease 2020:I5 2549-2558

2549

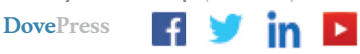

http:/doiorg/ $10.21471 \mathrm{COPD}, \mathrm{S} 269$. 
(ie, on waking up and in the first hours of the morning, until 11 a.m.), either just in one period or two or all of them. ${ }^{1}$

Along with the day, chest tightness, wheezing, cough, and phlegm and mainly chronic dyspnea can heterogeneously affect COPD patients according to the prevalence of the underlying disease (ie, fibrosing chronic bronchiolitis, with or without chronic bronchitis, pulmonary emphysema, overlap with asthma). ${ }^{2,3}$

Their occurrence during the night-time and at early morning, however, has been reported much more frequently than expected, not only in the presence of severe but also mild-to-moderate airflow obstruction ${ }^{4}$ and more importantly, has been related to adverse outcomes including worse quality of life, increased rate of COPD exacerbations and hospitalization and increment of all-cause mortality. ${ }^{5}$ Actually, under these circumstances, the most frequent, disabling, and prognostic symptom in COPD patients is dyspnea that could be related to the supine position.

Normally, when lying down because of gravity-related mechanical reasons, the functional residual capacity decreases, and inspiratory capacity specularly increases, despite a small reduction of total lung capacity. This can be limited or prevented if in COPD patients with moderate-to-severe, and very severe airflow obstruction tidal expiratory flow limitation (EFL) develops in the supine position, promoting postural dynamic pulmonary hyperinflation and its consequences. Among those, dyspnea might occur as a result of neuro-mechanical dissociation and increased (threshold) elastic work of breathing due to the intrinsic PEEP.

Therefore, this study aimed to assess the presence of supine tidal EFL and its possible relationship with the dyspnea occurrence reported during the night (orthopnea) and in the early morning from stable COPD patients and to see if there were useful tools to foresee this condition and possibly to prevent or reduce it.

\section{Methods}

\section{Subjects}

This study was performed at the Respiratory Medicine Unit of the University of Brescia - Spedali Civili Hospital of Brescia, Italy, from July 2018 to February 2019. To be included in the study the subjects, older than 45 yrs have had a diagnosis of COPD according to the presence of individual/environmental risk factors, typical chronic symptoms, and baseline spirometry, showing a not reversible obstructive ventilatory defect with $\mathrm{FEV}_{1} / \mathrm{VC}$ ratio lower than LLN. $^{6}$ The FEV1 value established the severity of airflow obstruction as $\%$ predicted. $^{7}$

The greatest possible attention was placed to exclude any relevant comorbidities potentially able to sustain similar symptoms such as chronic heart failure, cardiac arrhythmias, neoplasms, sleep-disordered breathing, obesity, asthma coexistence, endocrine diseases, prostatic hypertrophy, neuromuscular diseases or skeletal abnormalities involving particularly respiratory muscles and chest wall structure. Excluded were also subjects with neurocognitive disorders and those unable to perform correctly respiratory function tests.

The subjects could be smokers or ex-smokers and were functionally evaluated after adequate wash-out of bronchodilator drugs, at least 3 months after an eventual COPD exacerbation.

\section{Study Design}

In the morning, subjects enrolled in the study were asked to undergo respiratory function tests in sitting position wearing a nose-clip and breathing through a flanged mouthpiece to determine slow vital capacity (VC), inspiratory capacity (IC), forced vital capacity (FVC), forced expiratory lung volume in the first second $\left(\mathrm{FEV}_{1}\right)$, maximal expiratory flow-rates (FEFs) at different lung volumes through bell spirometry (BIOMEDIN Instruments, Padua, Italy). After few minutes, not directly measurable lung volumes, namely residual volume (RV) functional residual capacity (FRC), total lung capacity (TLC) were obtained by a constant pressure body plethysmograph (BIOMEDIN Instruments, Padua, Italy). During the procedure, patients panted at a 0.7 Hertz frequency. Alveolar Volume $\left(\mathrm{V}_{\mathrm{A}}\right)$ and Transfer Factor for CO (KCO) were also measured with a single breath method, and Lung Diffusion Capacity for CO (DLCO) subsequently obtained (BIOMEDIN Instruments, Padua, Italy).

Twenty minutes after that, tidal expiratory flow limitation (EFL) at rest was assessed in seated position and 10-15 minutes after recumbency by both the NEP method and FOT technique. ${ }^{8,9}$ Soon after that, always in the supine position, IC was measured to determine the absolute and percent changes as compared to IC in seated position, assuming that a decrease, no increase or an increase of IC less than $10 \%$ and $200 \mathrm{~mL}$ when patients were lying down meant the development of postural dynamic pulmonary hyperinflation (DH). This limit was empirically chosen because the opposite is considered as a 
significant decrease in dynamic hyperinflation after bronchodilator in COPD. ${ }^{10}$

For each functional parameter, the best value obtained from at least 3 acceptable and repeatable maneuvers were retained for analysis, except for lung diffusion measurements where only 2 tests were done.

All tests were performed according to the ERS-ATS recommendations. ${ }^{6}$ Predicted values of lung function parameters were those proposed by the European Community for Coal and Steel. ${ }^{11}$

Afterward, in the same day, in each patient, the impact of COPD on their life was measured by the COPD assessment test (CAT) administration, the subjective level of chronic dyspnea was obtained by modified Medical Research Council (mMRC) scale, and the Glasgow Depression Scale and Glasgow Anxiety Scale questionnaires were also used to determine the depression and anxiety risk.

Finally, a questionnaire to record symptoms during the night-time, at early morning (ie, on waking up and in the early morning until 11 a.m.) and in day-time was administered to each subject to investigate the prevalence and severity of different symptoms in these 3 different periods of the day in order to assess their variability in our cohort of stable COPD patients treated with standard therapy. The questionnaire we used was that adopted in the ASSESS (observAtional study on characterization of 24h Symptoms in chronic obstructivE pulmonary diSeaSe patient) study consisting of 33 questions aimed to detect the presence and distribution of the five main COPD-related symptoms throughout the previous week. In more detail, there were 13 questions for night-time symptoms (from to go to bed to the getting out of the bed), 10 questions for early morning symptoms (from the getting out of bed to the 11 a.m.) and 10 questions for day-time symptoms (from 11 a.m. to go to bed). ${ }^{12}$

Moreover, the questionnaire had some questions related to sleep-related disturbances (ie, difficulty in getting asleep, presence of nocturnal awakenings, body position assumed to favor sleep, ie, orthopnea), and to use of rescue drugs to alleviate symptoms in different parts of the day. All questionnaires were administered under the supervision of a trained physician.

The study was performed in accordance with the Helsinki declaration. The Ethic Committee of Spedali Civili University Hospital of Brescia approved the protocol of the study, and all patients provided written informed consent upon enrolling.

\section{Statistics}

Unless specified otherwise, data are expressed as the mean \pm standard deviation.

The Student's unpaired $t$-test was used to assess the difference of variables between COPD patients with or without supine EFL. The Exact Fischer test was used for comparing data relative to symptoms between COPD patients with or without supine tidal EFL and with or without significant IC changes in supine as compared to the seated position.

Statistical significance was accepted if $p \leq 0.05$. Statistical analyses were performed using Graph Pad Prism 6.0 (Graph Pad Software, La Jolla, CA) and SPSS 23.00 (IBM, Armonk, NY)

\section{Results}

Forty-two stable COPD patients with moderate-to-severe airflow obstruction and without relevant comorbidities were consecutively enrolled in the study.

Anthropometric and clinical data of the patients are shown in Table 1, all together and divided into 2 groups according to the absence (NFL) or presence (FL) of supine tidal EFL. No significant differences emerged between the 2 groups for demographic characteristics, but FL patients had more severe chronic dyspnea (by mMRC score) and perceived worse health status (by CAT score), albeit not significantly, than the NFL group. No different smoking habits emerged between NFL and FL groups. No differences were found concerning either anxiety or depression scores between FL and NFL subgroups.

Baseline respiratory function data are illustrated in Table 2, again in all patients, and divided into NFL and FL groups. COPD patients with supine EFL were more obstructed (lower $\mathrm{FEV}_{1} / \mathrm{VC}$ ratio and $\mathrm{FEV}_{1}$ ) with reduced $\mathrm{VC}$ as compared to COPD patients without supine EFL, but no significant differences in lung volumes and DLCO and $\mathrm{KCO}$ were observed.

IC was slightly lower in seated position but markedly reduced in supine position in FL COPD patients as compared with NFL COPD patients and, more importantly, in average IC did not show any significant change during recumbency, instead of normally increasing as observed in NFL COPD patients.

In detail, 21 out of 25 (84\%) FL COPD patients had an IC increase less than $10 \%$, while all NFL COPD patients had an IC increase equal or greater than $10 \%$, moving from seated to the supine position $(\mathrm{p}<0.001)$ (Figure 1). 
Table I Anthropometric Data of Subjects

\begin{tabular}{|c|c|c|c|c|}
\hline & ALL & NFL & FL & $\mathbf{p}$ \\
\hline Subjects (nr) & 42 & 17 & 25 & ns \\
\hline Age $(y r)$ & $65.2 \pm 8.7$ & $6.43 \pm 9.8$ & $66.4 \pm 7.9$ & ns \\
\hline Sex $(f / m)$ & ||$/ 3 \mid$ & $4 / 13$ & $7 / 18$ & ns \\
\hline Weight (kg) & $68.4 \pm 12.3$ & $68.9 \pm 15.1$ & $68.1 \pm 10.4$ & ns \\
\hline Height $(\mathrm{cm})$ & $168.7 \pm 7.6$ & $169.8 \pm 8.0$ & $167.9 \pm 7.4$ & ns \\
\hline BMI (kg/m2) & $24.0 \pm 3.4$ & $23.7 \pm 3.6$ & $24.1 \pm 3.3$ & ns \\
\hline Smoking habit (ExS/S) & $24 / 18$ & $7 / 10$ & $17 / 8$ & ns \\
\hline MRC (score) & $2.0 \pm 1.1$ & $1.4 \pm 0.9$ & $2.4 \pm 1.1$ & $P<0.004$ \\
\hline $0-1$ (\%) & $38.1(16)$ & $53.0(9)$ & $28.0(7)$ & \\
\hline 2 or $>(\%)$ & $61.9(26)$ & $47.0(8)$ & $72.0(18)$ & \\
\hline CAT (score) & $14.34 \pm 5.38$ & $12.6 \pm 4.8$ & $15.5 \pm 5.6$ & ns \\
\hline $0-15(\%)$ & $50.0(21)$ & $64.7(11)$ & $40.0(10)$ & \\
\hline$>15(\%)$ & $50.0(21)$ & $35.3(6)$ & $60.0(15)$ & \\
\hline GDS (score) & $7.68 \pm 4.79$ & $7.5 \pm 4.6$ & $7.8 \pm 5.0$ & ns \\
\hline GAS (score) & $14.34 \pm 5.38$ & $12.6 \pm 4.8$ & $15.5 \pm 5.6$ & ns \\
\hline \multicolumn{5}{|l|}{ Baseline COPD drugs } \\
\hline LAMA n(\%) & $4(10 \%)$ & $3(18 \%)$ & I (4\%) & \\
\hline LAMA+LABA n(\%) & II (26\%) & $4(23 \%)$ & $7(28 \%)$ & \\
\hline ICS+LABA n(\%) & $8(19 \%)$ & $3(18 \%)$ & $5(20 \%)$ & \\
\hline ICS+LABA+LAMA n(\%) & $19(45 \%)$ & 7 (4I\%) & $12(48 \%)$ & \\
\hline
\end{tabular}

Note: Data are mean \pm SD.

Abbreviations: ExS, ex-smokers; S, smokers.

Table 2 Baseline Functional Characteristics of Patients

\begin{tabular}{|c|c|c|c|c|}
\hline & ALL & NFL & $\mathbf{F L}$ & $\mathbf{p}$ \\
\hline VC (L) & $3.6 \pm 1.0$ & $4.0 \pm 0.9$ & $3.3 \pm 0.9$ & \\
\hline VC \% pred & $98.1 \pm 18.7$ & $105.7 \pm 15.1$ & $93.0 \pm 19.3$ & $\mathrm{p}<0.05$ \\
\hline FEVI/VC \% & $41.8 \pm 13.5$ & $47.8 \pm 10.0$ & $37.7 \pm \mid 4.2$ & \\
\hline FEVI/VC \% pred & $55.1 \pm 17.6$ & $62.7 \pm 12.5$ & $49.9 \pm 18.9$ & $\mathrm{p}<0.05$ \\
\hline FEVI (L) & $1.5 \pm 0.6$ & $1.9 \pm 0.5$ & $1.2 \pm 0.5$ & \\
\hline FEVI \% pred & $54.0 \pm 19.3$ & $65.1 \pm 12.0$ & $46.4 \pm 18.9$ & $\mathrm{p}<0.0 \mathrm{I}$ \\
\hline FRC (L) & $4.7 \pm 1.1$ & $4.7 \pm 1.1$ & $4.8 \pm 1.2$ & \\
\hline FRC \% pred & $142.9 \pm 29.2$ & $138.5 \pm 22.5$ & $|46.0 \pm 33|$. & ns \\
\hline $\mathrm{RV}(\mathrm{L})$ & $3.7 \pm 1.0$ & $3.5 \pm 0.9$ & $3.9 \pm 1.0$ & \\
\hline RV \% pred & $161.6 \pm 42.9$ & $151.0 \pm 34.2$ & $168.9 \pm 47.2$ & ns \\
\hline TLC (L) & $7.3 \pm 1.6$ & $7.5 \pm 1.6$ & $7.2 \pm 1.6$ & \\
\hline TLC \% pred & $118.8 \pm 19.2$ & $119.2 \pm 16.4$ & $118.6 \pm 21.3$ & ns \\
\hline TLCO ml/(min*mmhg) & $12.8 \pm 6.3$ & $14.8 \pm 7.0$ & $1 \mathrm{I} .3 \pm 5.4$ & \\
\hline TLCO \% pred & $51.7 \pm 23.5$ & $57.6 \pm 24.4$ & $47.4 \pm 22.4$ & ns \\
\hline $\mathrm{KCO} \mathrm{ml} /\left(\mathrm{min}^{*} \mathrm{mmhg}\right) / \mathrm{L}$ & $2.3 \pm 1.1$ & $2.5 \pm 1.2$ & $2.2 \pm 1.0$ & \\
\hline KCO \% pred & $55.4 \pm 24.7$ & $58.5 \pm 23.6$ & $53.1 \pm 24.7$ & ns \\
\hline IC (L) & $2.3 \pm 0.6$ & $2.6 \pm 0.6$ & $2.2 \pm 0.6$ & \\
\hline IC \% pred & $84.5 \pm 16.4$ & $88.8 \pm 12.2$ & $81.4 \pm 18.5$ & ns \\
\hline IC sup. \% pred & $92.5 \pm 21.5$ & $104.8 \pm 16.8$ & $83.8 \pm 20.5$ & $\mathrm{p}<0.0 \mathrm{I}$ \\
\hline$\Delta \mathrm{IC}(\mathrm{L})$ & $0.2 \pm 0.3$ & $0.39 \pm 0.28$ & $0.08 \pm 0.18$ & $\mathrm{p}<0.0 \mathrm{I}$ \\
\hline
\end{tabular}

Notes: Data are mean \pm SD.

Abbreviation: $\triangle \mathrm{IC}, \mathrm{IC}$ sup - IC.

During the night-time, 8 out of 25 (32\%) FL COPD patients had dyspnea at least 3 times in the past week as compared to 1 out of $17(6 \%)$ NFL COPD patients $(p=$
$0.06)$. Chest tightness and wheezing were reported only by FL COPD patients, in $3(12 \%)$ and $5(20 \%)$ out of 25 , respectively. Always during the night-time, cough and 


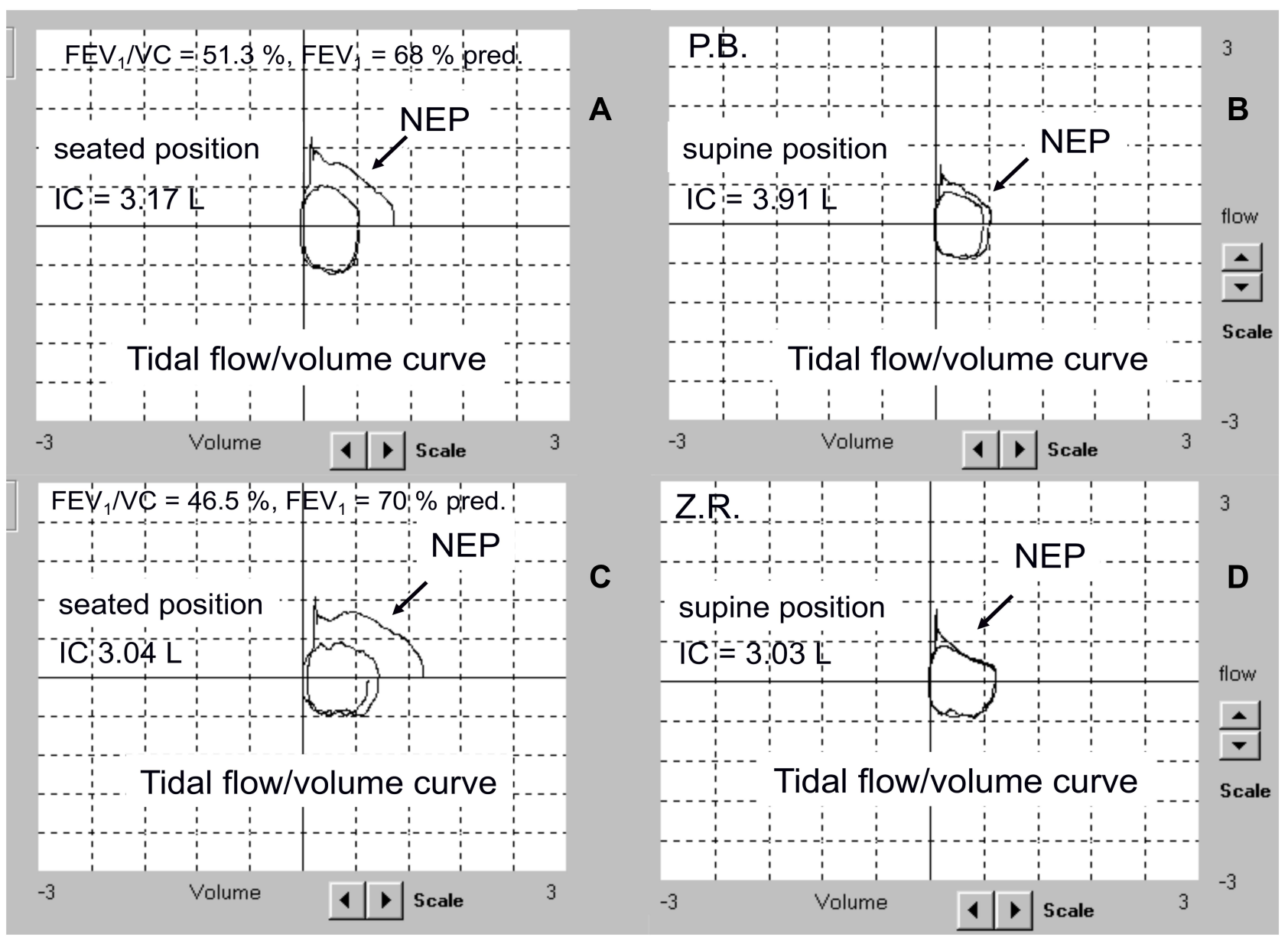

Figure I A representative COPD patient reporting no dyspnea and orthopnea during night-time and dyspnea at early morning (two days in the past week - no rescue drugs). Note the absence of supine EFL during tidal expiration assessed by the NEP method and an increase of supine IC of $730 \mathrm{~mL}$ (upper panels $\mathbf{A}$ and $\mathbf{B}$ ). A representative COPD patient reporting dyspnea and orthopnea during night-time and dyspnea at early morning (all days in the past week - rescue drugs on two occasions). Note the presence of supine EFL during tidal expiration assessed by the NEP method and no change in supine IC (lower panels $\mathbf{C}$ and $\mathbf{D}$ ).

phlegm were present at least 3 times in the past week in 13 out of $25(52 \%)$ FL COPD patients and in 3 out of 17 $(18 \%)$ NFL COPD patients $(\mathrm{p}<0.05)$ (Figure 2A).

Upon awakening and in the early morning, 17 out of 25 (68\%) FL COPD patients had dyspnea at least 3 times in the past week as compared to 4 out of 17 (23\%) NFL COPD patients $(\mathrm{p}<0.05)$. Again, chest tightness and wheezing were reported only by FL COPD patients, in 5 $(20 \%)$ and $2(8 \%)$ over 25, respectively. Always upon awakening and in the early morning, cough and phlegm were present at least 3 times in the past week in 11 out of 25 (44\%) FL COPD patients and in 10 out of $17(59 \%)$ NFL COPD patients (n. s.) (Figure 2B).

Dyspnea (during night-time and at early morning) was reported by $81 \%$ (17 out of 21 ) of COPD patients with an IC increase less than $10 \%$ of the respective seated IC value as compared to $33 \%$ (7 out of 21 ) of those with an IC increase $\geq 10 \%$ of the respective seated IC value $(p<0.05)$, suggesting postural $\mathrm{DH}$ as a main pathophysiological mechanism of this symptom (Figure 3). Three out of 7 patients claiming dyspnea during the night and/or in the early morning with significant supine IC increase were FL when lying down.

Concerning the sleep-related disturbances, 17 over 25 (72\%) FL COPD patients reported at least one among difficulty falling asleep, orthopnea after falling asleep and nocturnal awakenings, as compared to 3 out of 17 (18\%) NFL COPD patients more than twice in the past week $(\mathrm{p}<0.05)$.

In detail, difficulty falling asleep more than twice in the past week occurred in 7 out of 25 (28\%) FL COPD patients as compared to 1 out of $17(6 \%)$ NFL COPD patient, orthopnea was present only in FL COPD patients, namely in 7 out of 25 (28\%), and nocturnal awakenings were reported in 11 out of 25 (44\%) FL COPD patients versus 2 out of $17(12 \%)$ NFL COPD patients $(\mathrm{p}<0.05)$, respectively (Figure 4 ). 


\section{A \\ Night time symptoms}

(at least 3 nights in the past week)

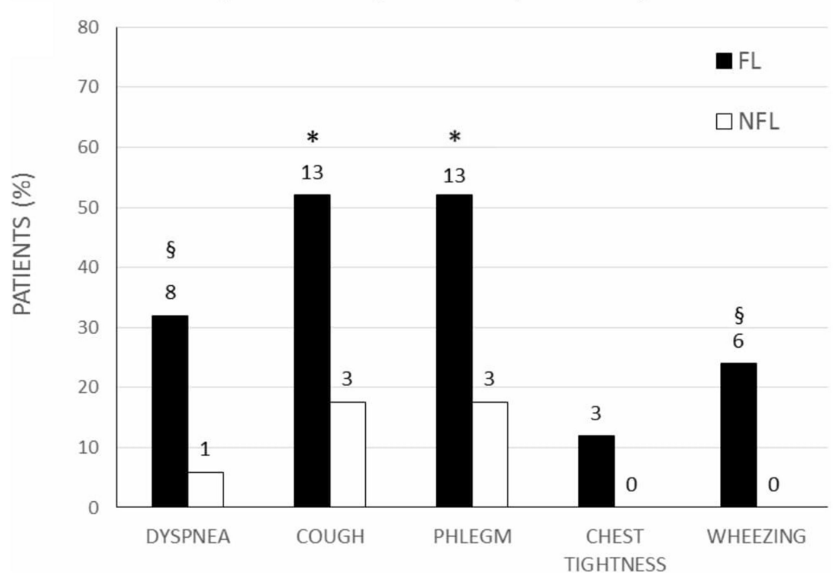

B

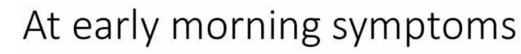

(at least 3 mornings in the past week)

Figure 2 (A) Night-time symptoms reported by FL and NFL COPD patients 3 or more times in the past week were shown. Cough and phlegm were more significantly reported in $\mathrm{FL}$ patients $\left({ }^{*} \mathrm{p}<0.05\right)$. Dyspnea occurred more frequently $(\$ \mathrm{p}=0.06)$ in $\mathrm{FL}$ patients as well as wheezing $(\$ \mathrm{p}=0.06)$. (B) At early morning symptoms reported by $\mathrm{FL}$ vs NFL COPD patients, 3 or more times in the past week were shown. Dyspnea was significantly more frequent in FL patients $\left({ }^{*} p<0.05\right)$.

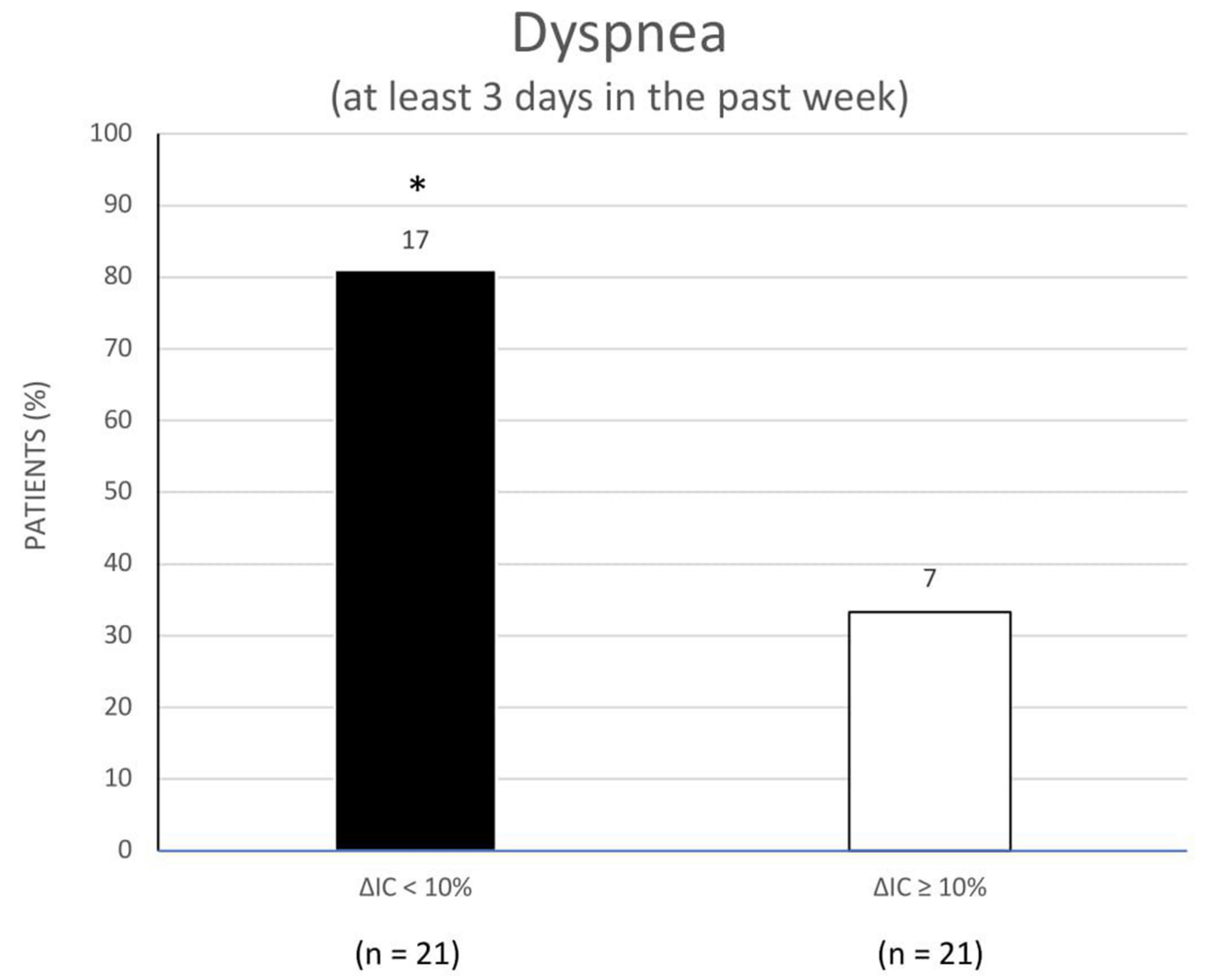

Figure 3 The presence of dyspnea reported, either during night-time or early morning, in the past week is shown in the function of change in IC with recumbency ( $\Delta \mathrm{IC}$ ). An increase of $\Delta \mathrm{IC}<10 \%$ of the respective value in seated position was associated with a significantly greater prevalence of dyspnea in COPD patients $(* \mathrm{p}<0.05)$.

\section{Discussion}

The main finding of this study is that dyspnea during night-time and/or at early morning is reported mostly or almost exclusively from COPD patients who exhibit tidal EFL in supine position, likely because this mechanical condition is very often associated with $\mathrm{DH}$, that is heralded by the lack of a significant IC increment with recumbency. ${ }^{13}$

While determining tidal EFL in the supine position might be problematic without negative expiratory pressure 


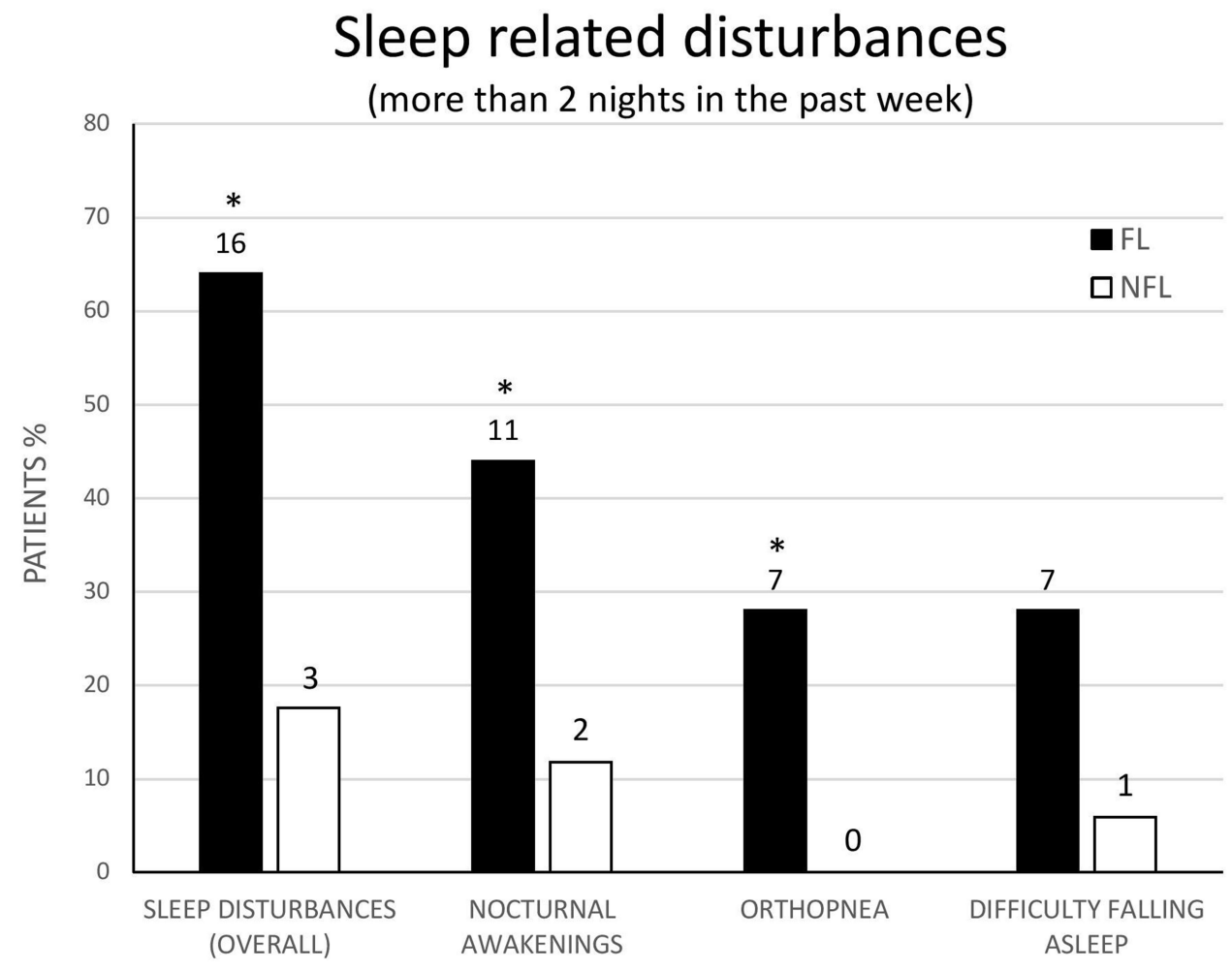

Figure 4 Sleep-related disturbances reported by FL and NFL COPD patients more than twice in the past week are shown. Overall, and for orthopnea, the frequency is significantly increased in FL patients $\left({ }^{*} \mathrm{p}<0.05\right)$.

(NEP) devices or FOT availability, the measurement of IC at rest can be very easily obtained in both positions (seated and supine) in any Lab, suggesting the development of supine DH if IC does not increase or increases very little when the patient is lying down and giving a pathophysiologic explanation of his/her dyspnea occurring during the night-time or at early morning. In this respect, no or minimum increment of IC with recumbency is more explanatory of dyspnea under these conditions than the presence of supine tidal EFL.

In fact, while essentially none of NFL COPD patients reported dyspnea during the night-time, and about one/ fourth in the early morning, possibly due to the beginning of the daily physical activity, not all FL COPD patients were complaining of dyspnea during the night-time (only one/third did) and/or at early morning (only two/third did). One reason is that resting EFL does not induce necessarily dynamic pulmonary hyperinflation if the expiratory time is long enough to deflate the lung until the relaxation volume of the respiratory system. Therefore, not all FL COPD patients (21 out of 25) do exhibit a lack or a trivial increase of supine IC. Another reason could be the threshold of supine DH needed to produce dyspnea, which is a function of the level of intrinsic PEEP (and related increase in work of breathing) produced, depending on pulmonary compliance at end-expiratory lung volume. Actually, not all FL COPD patients with absent IC increase declared persistent dyspnea during the night and/or at early morning (17 out of 21). Finally, for dyspnea to be perceived, the duration of supine $\mathrm{DH}$ might be important since dyspnea was reported in FL COPD patients by $32 \%$ during night-time and by $68 \%$ at early morning.

COPD-related chronic symptoms were recently addressed in the function of the period of the day in different cohorts of stable COPD patients, and a large variability was found in more than two/third of the patients. $^{2}$ Quite surprisingly, these symptoms also occurred during the night-time and at early morning in a large percentage of these patients and not only in those with severe or very severe airflow obstruction. ${ }^{1,3,4}$

Dyspnea during the night-time more than others chronic symptoms were related to worse prognosis in a large population of COPD patients followed for a mean of 5 years, predicting a higher risk of COPD-related exacerbation and hospitalization and all-cause mortality. ${ }^{5}$ This 
was true after adjusting for age, sex, and severity of airflow obstruction. In this general COPD population with a prevalence of mild and moderate GOLD stages, night-time dyspnea was initially reported from $4 \%$ of the patients and was related to the severity of airway obstruction, chronic dyspnea, phlegm and cough and presence of ischemic heart disease and atrial fibrillation. ${ }^{5}$

The pivotal ASSESS study was designed to characterize and determine the prevalence and severity of early morning, day and night-time symptoms and to evaluate their correlation with each other and with different aspects of COPD such as chronic dyspnea, disease severity, health-related quality of life, levels of anxiety/depression, sleep quality and COPD exacerbations. A cohort of 726 stable COPD patients, aged $67 \pm 9$ yrs with mean $\mathrm{FEV}_{1}=$ $53 \pm 21 \%$ pred., mostly in stage 2 and 3 GOLD, receiving the usual therapy, was recruited. The night-time and early morning symptoms were largely reported at the baseline visit. More than $50 \%$ of the patients declared dyspnea, cough, and phlegm three or more times in a typical week or the past week at early morning and more than $30 \%$ at the night-time, while lower percentages of patients complained by wheezing and chest tightness in these periods of the day. ${ }^{12}$ After 6 months of checking by a follow-up phone interview, the annualized number of COPD exacerbations were doubled in the COPD patients with nighttime and early morning symptoms as compared with those without them. ${ }^{14}$ However, although there was a significant association between night-time and early morning symptoms and exacerbations during follow-up, at multivariate analysis, the presence of the symptoms did not stay as an independent factor predicting exacerbations. ${ }^{14}$

In a survey performed by telephone interview in a quite large number of COPD patients $(n=1239)$, meeting the inclusion criteria of the study and agreed to participate, the respondents (about 60\%) who declared both night-time and early morning symptoms, had a greater probability of low or moderate adherence to COPD treatment and a significantly higher risk of COPD exacerbations (one or more) in the past year. ${ }^{15}$

Therefore, there is consistent evidence than night-time and early morning symptoms, mainly dyspnea and chronic cough and phlegm, are associated with the greater risk of having future COPD exacerbation, particularly if not adequately treated. ${ }^{5,14,15}$

Sleep disturbances were also mostly reported from FL COPD patients and significantly more than NFL COPD patients. Low quality of sleep due to poor sleep efficiency and frequent symptom-related nocturnal awakenings has been inversely related to health status and more than chronic dyspnea or degree of airflow obstruction in COPD patients. ${ }^{16}$ In a cohort of 98 COPD patients with moderate-to-severe airflow obstruction, a series of insomnia-related symptoms, such as difficulty to fall asleep, nocturnal awakenings, adequacy of sleep duration were cross-sectionally related to dyspnea and cough, but not to $\mathrm{FEV}_{1}$ value. More interestingly, these insomnia-related symptoms were longitudinally related to a higher risk of COPD exacerbations and hospitalizations and all-cause mortality in 2.4 years mean follow up, also after adjusting for several confounders such as socio-demography characteristics, BMI, $\mathrm{FEV}_{1}$, and COPD global severity score. ${ }^{17}$

In our cohort of patients, orthopnea, defined as the necessity of assuming the seated position to breathe during the night, putting two or more pillows behind the back, was reported only by FL COPD patients. The presence of orthopnea was shown to occur much more frequently in FL COPD patients, as compared with NFL COPD patients already 20 years ago by Eltayara and coworkers in a series of 98 stable COPD patients without cardiovascular comorbidities. ${ }^{18}$ Our findings confirm this previous observation, indicating that nocturnal orthopnea in COPD patients without relevant comorbidities is associated with supine tidal EFL.

Ultimately, the occurrence of COPD-related symptoms during night-time and at early morning needs to be carefully assessed in COPD patients, also in those with mildto-moderate airflow obstruction, because their presence is likely associated not only with worse quality of life but also with adverse outcomes in COPD patients. Although in the presence of frequent phlegm and cough, suggesting the coexistence of chronic bronchitis or less frequently bronchiectasis, a greater possibility of negative events such as COPD-related exacerbations and hospitalizations has been already demonstrated together faster lung function decline in COPD patients, ${ }^{19-21}$ also resting dyspnea during night-time and/or at early morning has been associated with deleterious consequences such as sleep disturbances, severe acute exacerbations and all-cause mortality in COPD patients. ${ }^{5,14,15,17}$

Based on our findings, a mechanistic link between dyspnea and the aforementioned clinical consequences under these circumstances is very often the persistent development of $\mathrm{DH}$ during recumbency that is greatly favored by the presence of supine EFL. Normally, when lying down, tidal breathing moves at lower lung volume 
with FRC shifting towards RV, and IC substantially increases. This largely happens even in COPD patients with mild-to-moderate airflow obstruction. Sometimes this shift does not occur or is limited because the expiratory flow reserve towards RV is too scarce, and tidal EFL is unavoidable in COPD patients with greater airflow obstruction. $^{22}$ As a functional consequence, a postural DH may develop, and this can be easily shown by an absent or trivial increase of IC in the supine position as compared with the seated position. Since DH can induce dyspnea by several mechanisms, we suggest measuring the recumbent IC change in COPD patients claiming frequent orthopnea and/or dyspnea at early morning without other possible reasons. $^{23}$

In case any effort should be done to obtain round-theclock symptom control, particularly dyspnea, treating these COPD patients with a bronchodilator drug regimen that maintains them deflated as much as possible also during the second half of the day. ${ }^{24}$

We recognize that the relatively small number of COPD patients is a limit of the study, as well as the reliability of the questionnaires to assess the presence of symptoms accurately, but this is what usually has been used in similar studies. Moreover, the presence of a control group could have been useful to have a more informative comparison.

The strength of our work is to have selected COPD patients very carefully without confounding comorbidities that in the presence of moderate-to-severe airflow obstruction is a difficult task, so limiting the cohort of the patients that could be enrolled in the study.

In conclusion, we found that in stable COPD patients without comorbidities, dyspnea during the night-time and/ or at early morning is strongly associated with the presence of supine EFL, frequently leading to the development of postural $\mathrm{DH}$, as shown by the absence of significant IC increase during recumbency. Thus, no or trivial increase in supine IC may be a useful functional biomarker to foresee dyspnea under these conditions.

\section{Acknowledgments}

The Authors acknowledge Mr. M. Guerini, Lab. technician, for his invaluable technical support.

\section{Author Contributions}

All authors made substantial contributions to conception and design, acquisition of data, or analysis and interpretation of data; took part in drafting the article or revising it critically for important intellectual content; agreed to submit to the current journal; gave final approval of the version to be published; and agree to be accountable for all aspects of the work.

\section{Disclosure}

The authors report no conflicts of interest for this work.

\section{References}

1. Partridge MR, Karlsson N, Small IR. Patient insight into the impact of chronic obstructive pulmonary disease in the morning: an internet survey. COPD requires round-the-clock symptom control. Curr Med Respir Opin. 2009;25(8):2043-2048. doi:10.1185/030079909031 03006

2. Kessler R, Partridge MR, Miravitlles M, et al. Symptom variability in COPD. Eur Respir J. 2011;37(2):264-272. doi:10.1183/09031936. 00051110

3. Lopez-Campos JL, Calero C, Quintana-Gallego E. Symptom variability in COPD: a narrative review. Int J Chron Obstruct Pulmon Dis. 2013;8:231-238. doi:10.2147/COPD.S42866

4. Agusti A, Hedner J, Marin JM, Barbè F, Cazzola M, Rennard S. Night time symptoms: a forgotten dimension of COPD. Eur Respir Rev. 2011;20(121):183-194.

5. Lange P, Marott JL, Vestbo J, Nordestgaard BG. Prevalence of nighttime dyspnea in COPD and its implication for prognosis. Eur Respir J. 2014;43(6):1590-1598. doi:10.1183/09031936.00196713

6. Miller MR, Crapo R, Hankinson J, et al. General considerations for lung function testing. Eur Respir J. 2005;26(1):1533-1611. doi:10.1183/09031936.05.00034505

7. Global strategy for the diagnosis, management, and prevention of chronic obstructive pulmonary disease. NHLBI/WHO Global Initiative for Chronic Obstructive Lung Disease (GOLD) workshop summary (report-2017). Available from: http://www.goldcopd.org.

8. Koulouris NG, Valta P, Lavoie A, et al. A simple method to detect expiratory flow limitation during spontaneous breathing. Eur Respir J. 1995;8(2):306-313. doi:10.1183/09031936.95.08020306

9. Dellacà RL, Santus P, Aliverti A, et al. Detection of expiratory flow limitation using the forced oscillation technique. Eur Respir J. 2004;23(2):232-240. doi:10.1183/09031936.04.00046804

10. Newton MF, O’Donnell DE, Forkert I. Response of lung volume to inhaled salbutamol in a large population of patients with severe hyperinflation. Chest. 2002;121(4):1042-1050. doi:10.1378/ chest.121.4.1042

11. Quanjer PH, Tammeling GJ, Cotes JE, Pedersen OF, Peslin R, Yernault JC. Lung volumes and forced ventilatory flows. Eur Respir J. 1993;6(Suppl 16):5-40. doi:10.1183/09041950.005s1693

12. Miravitlles M, Worth H, Soler Cataluna JJ, et al. Observational study to characterize 24-hour COPD symptoms and their relationship with patient-reported outcomes: results from the ASSESS study. Respir Res. 2014;15(1):122-135. doi:10.1186/s12931-014-0122-1

13. Tantucci C. Expiratory flow limitation: definition, mechanisms, methods, and significance. Pulm Med. 2013;2013:749860. doi:10.1155/ 2013/749860

14. Miravitlles M, Worth H, Soler Cataluna JJ, et al. The relationship between 24-hour symptoms and COPD exacerbations and healthcare resource use: results from an observational study (ASSESS). Chronic Obstruct Pulm Dis. 2016;13(5):561-568.

15. Stephenson JJ, Cai Q, Mocarski M, Tan H, Doshi JA, Sullivan SD. Impact and factors associated with night-time and early morning symptoms among patients with chronic obstructive pulmonary disease. Int J Chron Obstruct Pulmon Dis. 2015;10:577-586. doi:10.2147/COPD.S76157 
16. Nunes DM, Mota RM, de Pontes Neto OL, Pereira ED, de Bruin VM, de Bruin PF. Impaired sleep reduces quality of life in chronic obstructive pulmonary disease. Lung. 2009;187(3):159-163. doi:10.1007/ s00408-009-9147-5

17. Omachi TA, Blanc PD, Claman DM, et al. Disturbed sleep among COPD patients is longitudinally associated with mortality and adverse COPD outcomes. Sleep Med. 2012;13:476-483. doi:10.1016/j.sleep.2011.12.007

18. Eltayara L, Ghezzo H, Milic-Emili J. Orthopnea and tidal expiratory flow limitation in patients with stable COPD. Chest. 2001;119(1):99104.

19. Kim V, Han MK, Vance GB, et al. The chronic bronchitis phenotype of COPD. An analysis of the COPD gene study. Chest. 2011;140 (3):626-633.

20. Vestbo J, Prescott E, Lange P. Association of chronic mucus hypersecretion with $\mathrm{FEV}_{1}$ decline and chronic obstructive pulmonary disease morbidity. Copenhagen City Heart Study Group. Am J Crit Care Med. 1998;153(5):1530-1535. doi:10.1164/ajrccm.153.5.8630597
21. Patel IS, Vlahos I, Wilkinson TM, et al. Bronchiectasis, exacerbation indices, and inflammation in chronic obstructive pulmonary disease. Am J Crit Care Med. 2004;170(4):400-407. doi:10.1164/rccm. 200305-6480C

22. Calverley PMA, Koulouris NG. Flow limitation and dynamic hyperinflation key concepts in modern respiratory physiology. Eur Respir J. 2006;25(1):186-199. doi:10.1183/09031936.04.00113204

23. Gagnon P, Guenette JA, Langer D, et al. Pathogenesis of hyperinflation in chronic obstructive pulmonary disease. Int J Chron Obstruct Pulmon Dis. 2014;9:187-201.

24. O’Donnell DE, Webb KA, Neder JA. Lung hyperinflation in COPD: applying physiology to clinical practice. Chronic Obstruct Pulm Dis. 2015;1(1):4-16.

\section{Publish your work in this journal}

The International Journal of COPD is an international, peer-reviewed journal of therapeutics and pharmacology focusing on concise rapid reporting of clinical studies and reviews in COPD. Special focus is given to the pathophysiological processes underlying the disease, intervention programs, patient focused education, and self management protocols. This journal is indexed on PubMed Central, MedLine and CAS. The manuscript management system is completely online and includes a very quick and fair peer-review system, which is all easy to use. Visit http://www.dovepress.com/testimonials.php to read real quotes from published authors. 